\title{
Enhancement of the transparency and flatness of engraved facets in a broadband range by optimizing its engraved structure.
}

\author{
I. Gurwich A*1, Y. Greenberg ${ }^{2}$, K. Harush ${ }^{3}$, and Y. Tzabari ${ }^{4}$ \\ ${ }^{1}$ Photonics and Electro-Optics Engineering Unit, Ben-Gurion University, Beer-Sheva \\ 8410501, Israel \\ ${ }^{2}$ Photonics and Electro-Optics Engineering Unit, Ben-Gurion University, Beer-Sheva \\ 8410501, Israel \\ ${ }^{3}$ Dep. Electrotechnics and Electronics, Academic College Sami Shamoon, Beer-Sheva \\ 8410802, Israel \\ ${ }^{4}$ Dep. Electrotechnics and Electronics, Academic College Sami Shamoon, Beer-Sheva \\ 8410802, Israel
}

August 27, 2020

\begin{abstract}
Achieving devices to be transparent is the task considered in the last time in many aspects and for different purposes. One issue of these problems is making anti-reflective surfaces in a wide wavelength range and keeping it flat enough. The authors of the previous publication showed that one could enhance a flat facet's transmission efficiency by suitable engraving. They used smoothed conical fingers and holes. Here, we widen the class of anti-reflective metasurfaces under consideration, following the requirements of the model developed in the previous paper. We involve also smoothed pyramidal fingers. The obtained results provide the improved engraved structure, with parameters dependent on the required spectral range, and the facet format. The predicted level of transmittance is close to $99 \%$, and the flatness(estimated by the standard deviation) as $0.2 \%$. This improvement is significant enough for high and broadband transmittance. In this work, we show that the randomization of the engraving parameters does not provide any significant effect for small facets. We also discuss a simple way of comparing experimental and theoretical results for a waveguide with the considered input and output features. In this study, as well as in our previous work, we restrict ourselves by rectangular facets. We also discuss the limitations that originated from the size and shape of the waveguide facets.
\end{abstract}

\section{Introduction}

We began the discussion on highly transparent waveguides in the previous publication ([1]). There, the general theoretical consideration of the engraved surfaces was given in the approximation of the single scattering (Born's) approximation. The result required the unit-cell to be elements possessing a constant slope, i.e., cones or pyramids (in the literature, other different shapes were discussed without preliminary justification [2]). The study in ([1]) concerns only conically shaped unit-cells. The existence of multiple scattering leads to the necessity of smoothing of cones to paraboloids. With this, there were notes that maximization of the fill-factor of the facets is not less important, and thus smoothed pyramidal structures promise to be more efficient. With the right choice of the conical unitcells structure parameters, one can get the transmission of a small size facet a little more than $98 \%$ and the flatness in the given wavelength range as $1 \%$.

*Corresponding Author: iosephg@gmail.com 


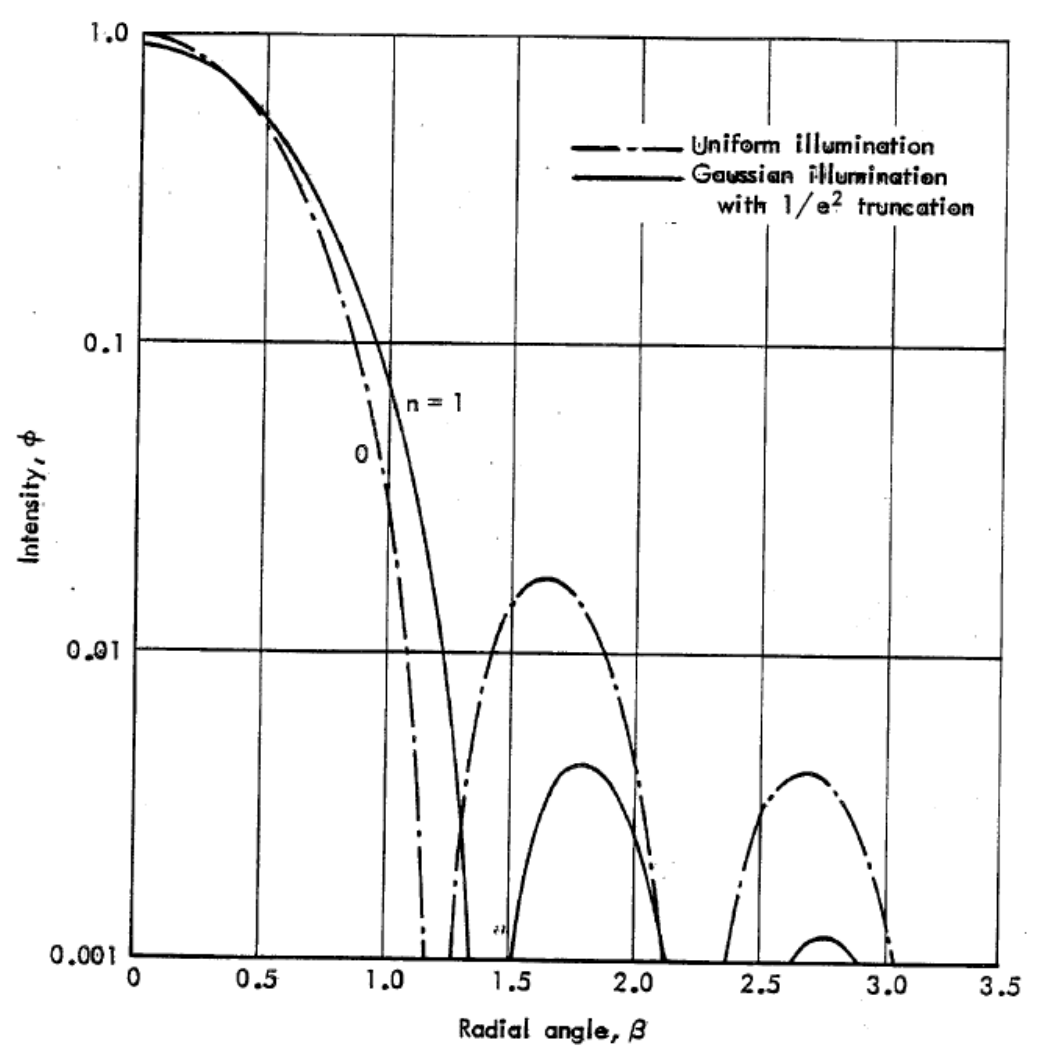

Figure 1: Reducing the side lobes of the beam diffracted by a circular hole by using the Gaussian shape.

We also have to note that the positive effect of randomization of the facet structure was discussed in the literature for a long time without reliable conclusions. Our remarks concerning the fill-factor suggest height-randomization of the unit-cells (but not the bottom size and position) in the structure to get possibly a higher and more uniform transmission for the broadband spectrum. But it is only preliminary expectations. All these states we tested numerically in the present study.

One can evaluate the anti-reflection efficiency of a surface structure from two different points of view. The first evaluation can be done for the structure itself, while used in the ideal condition: it is an engraved infinite surface illuminated by a plane wave.

Such structures can be approximated by a multilayered configuration, which describes the gradient index layer when the number of layers tends to infinite. The rigorous theoretical analysis of the response of this structure is given in Appendix A.

Another evaluation (more relevant for different applications) can be done for an actual facet of a particular shape and a finite square. With this, one has to pay attention to the geometrical parameters. In practical cases, we deal with a given size facet, illuminated by a specific beam. Therefore, a part of the energy can avoid the structured surface. With this, it contributes to the extra-reflection, measured in the experiment. Moreover, dealing with a nano-structured facet of a light guide, and using the chosen wavelength, we often cannot minimize the size of the focal spot of the input beam. We did not provide this analysis in the previous publication, taking in calculations only that part the beam power, which hits the facet. Reducing the edge effect requires additional means. This problem is analogous to one of reducing side-lobes of a beam diffracted by a hole. This is the old problem of classical optics. One of the conventional means, resolving it for a circular hole, is in using Gaussian beams. The proper illustration given below is imported from [[3]].

We can probe such a solution in our case as well. It means using the apodization of the whole structure. However, the simplified calculations (see Appendix B) show that:

- The angular distribution of the diffracted beam occurs to be partly smoothed by the fingers' parabolic shape, but not by an edge apodization;

- The critical angle for total internal reflection (TIR) in our case (waveguide material) allows to collect all forth-scattering peak. Thus, the edge effect is not significant at all; 


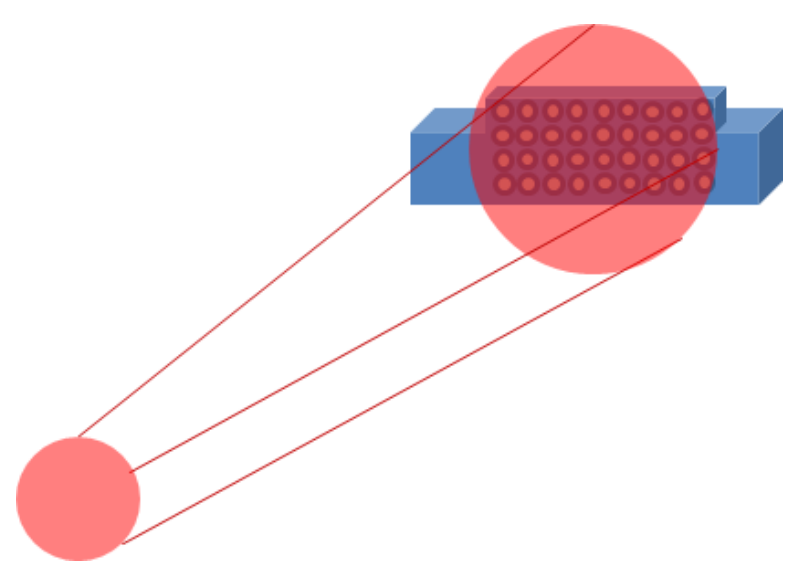

Figure 2: Illumination of a facet by a laser beam.

- Apodization leads to redistribution of the beam energy in favor to the side lobes. This will result in increasing the losses by those lobes, foe which the TIR condition will be broken.

Finally, the considering structures do not require any correction for diminishing the edge effects.

\section{Parabolic smoothing}

As was discussed in the previous part, the contribution of multiple scattering dictates us to modify the conic shape of the unit-cells obtained by minimizing the reflection (discussion on the contribution of multi-scattering effect see, e.g., [4],[5],[6]). Our previous publication showed that effective modification is in using a paraboloid shape instead of the proper cone. Here we test the efficiency of smoothing pyramidal unit-cells.

The advantage of parabolic smoothing is in obtaining the surface smooth also with respect to its derivatives (a higher order of smoothness).

Anyway, it is necessary to note that this recommendation cannot be accepted as the general one, equally efficient for any shape of the cross-section. Numerical tests are required for any specific configuration of the cross-section. The smoothing efficiency is tested here for round, square, rectangular, and hexagonal basis of unit-cells (fingers).

\section{Height Randomization of unit-cell in conic-parabolic struc- ture}

It is well known (see, e.g., [2]), that for a single spectral line one can obtain the almost zero reflection. Such a result is achieved without any randomization. However, any widening of the required spectral range affects the possible minimal reflection while understanding it in terms of the mean value. But this is yet not the well-defined formulation of the goal. And for a trustful evaluation of the role of randomization, one has to proceed from an explicit criterion. In the present discussion, we accept the following one:

- maximization of the mean value of transmission in the required spectral interval;

- additionally, the minimization of the standard deviation of transmission.

The previous discussions on effect of randomization one can find, e.g., in [7], [8]. However, first of all, we have to underline some significant aspects.

a) The true effect of randomization can be seen only for a large enough facet. Any statistics is valid only for a large number of elements (unit cells).

b) The optimal structure of the whole facet have to take into account the profile of the illuminating beam.

The illumination scheme in a virtual experiment (and namely this illumination - of the Gaussian shape - is taken into account in our numerical calculations) is depicted in Fig. 2

We see immediately, that this geometry does not respond to the aforementioned requirements. Therefore, the results presented below are not the best those, which could be obtained with the same 


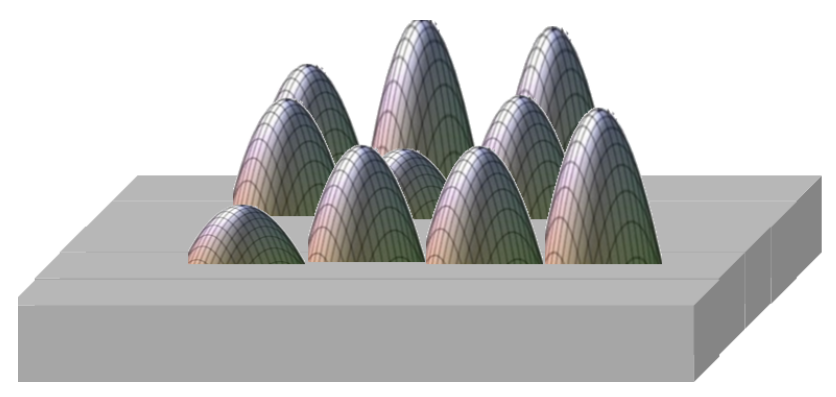

Figure 3: Randomized paraboloids structure

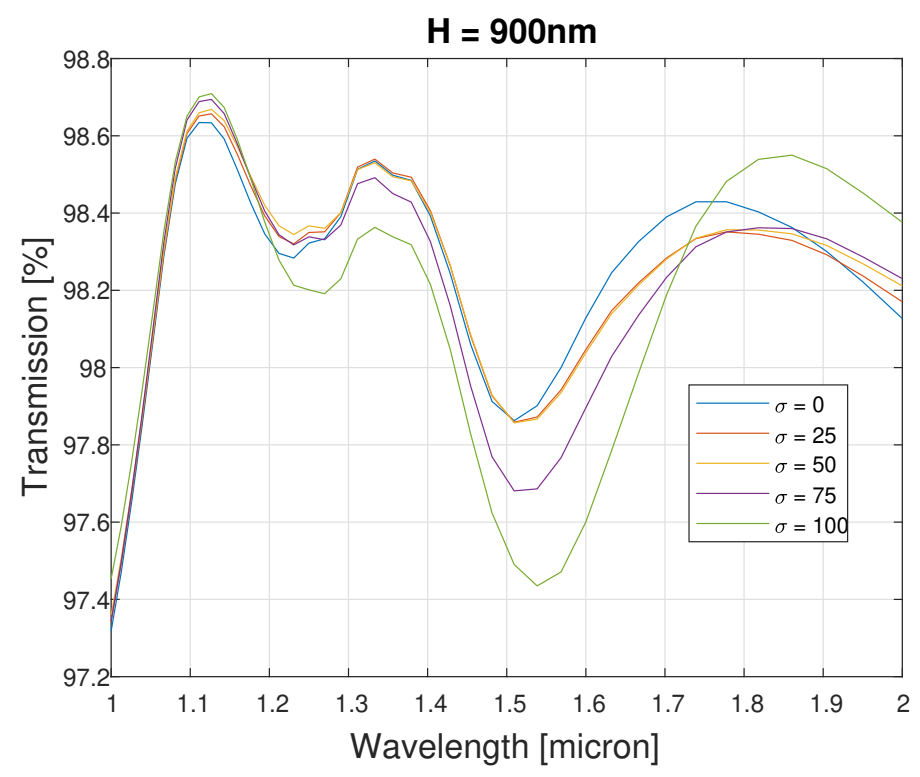

Figure 4: Transmission for paraboloid facet with the average height $H=900 \mathrm{~nm}$ and different levels of randomization.

finger-structure in the ideal conditions.

In the previous publication, we verbally discussed the possible effect of randomization of fingers' heights in the structure (see Fig. 3). Here we present the results of the numerical analysis, which show that the conclusions about the efficiency of the randomization are not straightforward.

Here, we recall the results concerning the effect of the height of conic, parabolic smoothed fingers on the transmittance of the facet.

Turn to the graphs depicting the transmission efficiency of the facets (of a small dimension, as 20x3 unit-cells) studded by parabolic fingers.

Here the value of the standard deviation $\sigma$ indicates the randomization level.

We calculated the parameters chosen as the criterion:

- For nonrandom structure $\left(\sigma_{H}=0\right)$ : the mean transmission is $T_{\text {mean }}=0.9825$, and the standard deviation of transmission is $\left(\sigma_{T}=0.003\right)$;

- For $\sigma_{H}=25 \mathrm{~nm}$ : $T_{\text {mean }}=0.98246$, and $\sigma_{T}=0.003009$;

- For $\sigma_{H}=50 \mathrm{~nm}: T_{\text {mean }}=0.98254$, and $\sigma_{T}=0.003011$

- For $\sigma_{H}=75 \mathrm{~nm}: T_{\text {mean }}=0.98217$, and $\sigma_{T}=0.003307$;

- For $\sigma_{H}=100 \mathrm{~nm}: T_{\text {mean }}=0.98184$, and $\sigma_{T}=0.003375$.

We see that for the given facet, it is not easy to conclude which one from the tested randomized structure is the best. The conclusion that begs here is: for small apertures, randomization is not efficient. And it is not a surprising result; it was already underlined above.

For supporting this statement, we present below additional graphs. 


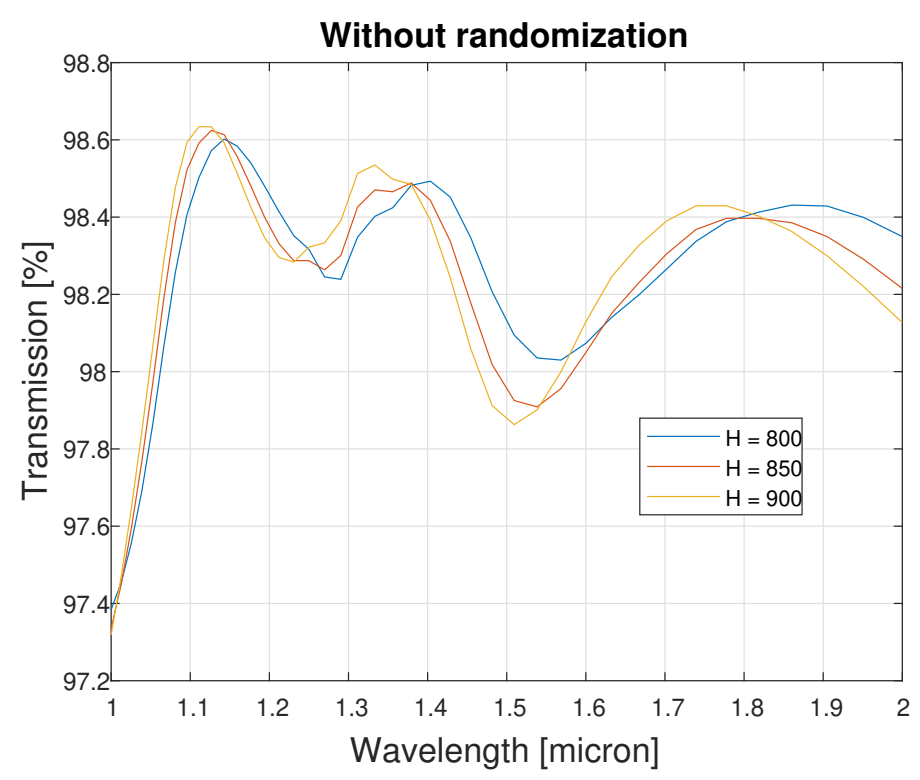

Figure 5: Transmissions for paraboloid facets without randomization.

- Without randomization:

$\mathrm{H}=800 \mathrm{~nm}$; thus $T_{\text {mean }}=0.9825$, and $\sigma_{T}=0.002925$;

$\mathrm{H}=850 \mathrm{~nm}$; thus $T_{\text {mean }}=0.982369$, and $\sigma_{T}=0.002969$;

$\mathrm{H}=900 \mathrm{~nm}$; thus $T_{\text {mean }}=0.9818$, and $\sigma_{T}=0.003$;

and statistical evaluations.

- With $\sigma_{H}=25 \mathrm{~nm}$ :

$\mathrm{H}=800 \mathrm{~nm} ; T_{\text {mean }}=0.98186$, and $\sigma_{T}=0.003174$;

$\mathrm{H}=850 \mathrm{~nm} ; \mathrm{T}_{\text {mean }}=0.98249$, and $\sigma_{T}=0.003209$;

$\mathrm{H}=900 \mathrm{~nm} ; T_{\text {mean }}=0.98246$, and $\sigma_{T}=0.003009$.

- With $\sigma_{H}=50 \mathrm{~nm}$ :

$\mathrm{H}=800 \mathrm{~nm} ; \mathrm{T}_{\text {mean }}=0.98014$, and $\sigma_{T}=0.003617$;

$\mathrm{H}=850 \mathrm{~nm} ; T_{\text {mean }}=0.98215$, and $\sigma_{T}=0.003129$;

$\mathrm{H}=900 \mathrm{~nm} ; T_{\text {mean }}=0.98254$, and $\sigma_{T}=0.003011$.

- With $\sigma_{H}=75 \mathrm{~nm}$ :

$\mathrm{H}=800 \mathrm{~nm} ; T_{\text {mean }}=0.978258$, and $\sigma_{T}=0.004591$;

$\mathrm{H}=850 \mathrm{~nm} ; T_{\text {mean }}=0.98194$, and $\sigma_{T}=0.003257$;

$\mathrm{H}=900 \mathrm{~nm} ; T_{\text {mean }}=0.982171$, and $\sigma_{T}=0.003307$.

- With $\sigma_{H}=100 \mathrm{~nm}$ :

$\mathrm{H}=800 \mathrm{~nm} ; T_{\text {mean }}=0.980236$, and $\sigma_{T}=0.00793$;

$\mathrm{H}=850 \mathrm{~nm} ; T_{\text {mean }}=0.980236$, and $\sigma_{T}=0.004047 ;$

$\mathrm{H}=900 \mathrm{~nm} ; T_{\text {mean }}=0.9801797$, and $\sigma_{T}=0.003775$.

However, some conclusions can still be made: a larger $\sigma_{H}$, requires a larger mean value of $H$. It is interesting, but the actual technology restricts a possible value of the height. Say, while using FIB, we face the restriction as $H<2 d_{b}$, where $d_{b}$ is the base diameter of the paraboloid. This fact puts an additional restriction on the ability and efficiency of randomization. 


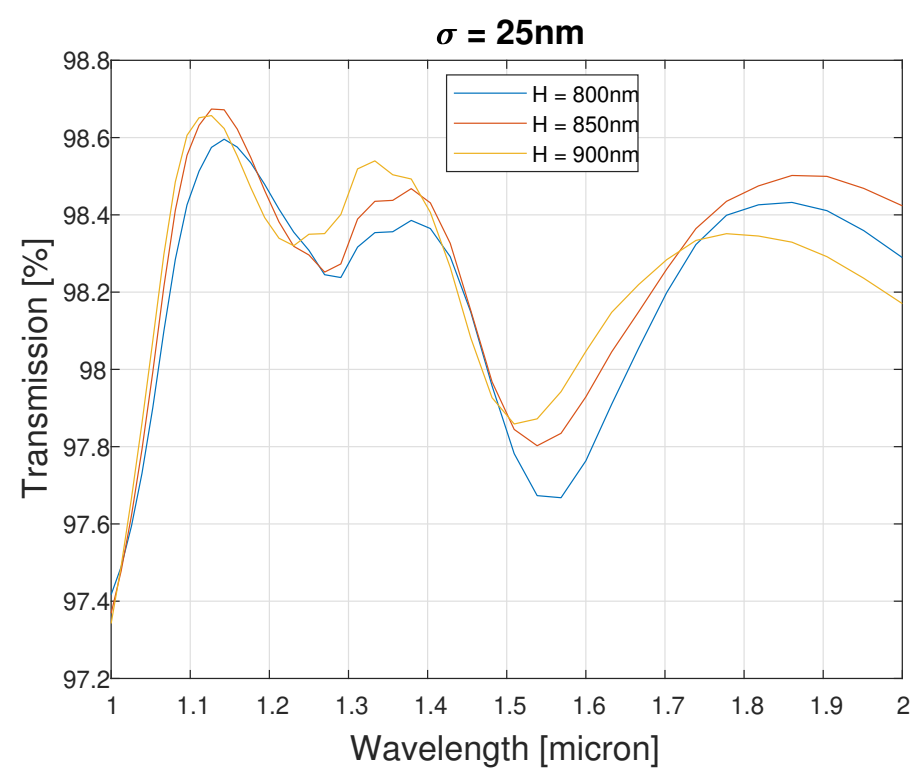

Figure 6: Transmissions for paraboloid facets with $\sigma_{H}=25 \mathrm{~nm}$.

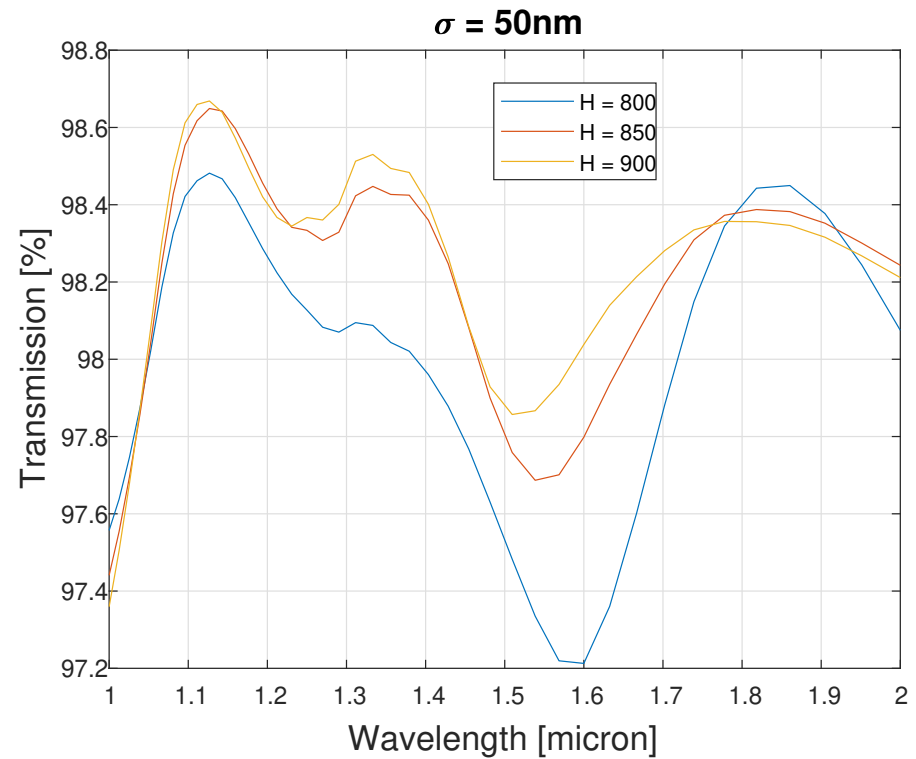

Figure 7: Transmissions for paraboloid facets with $\sigma_{H}=50 \mathrm{~nm}$. 


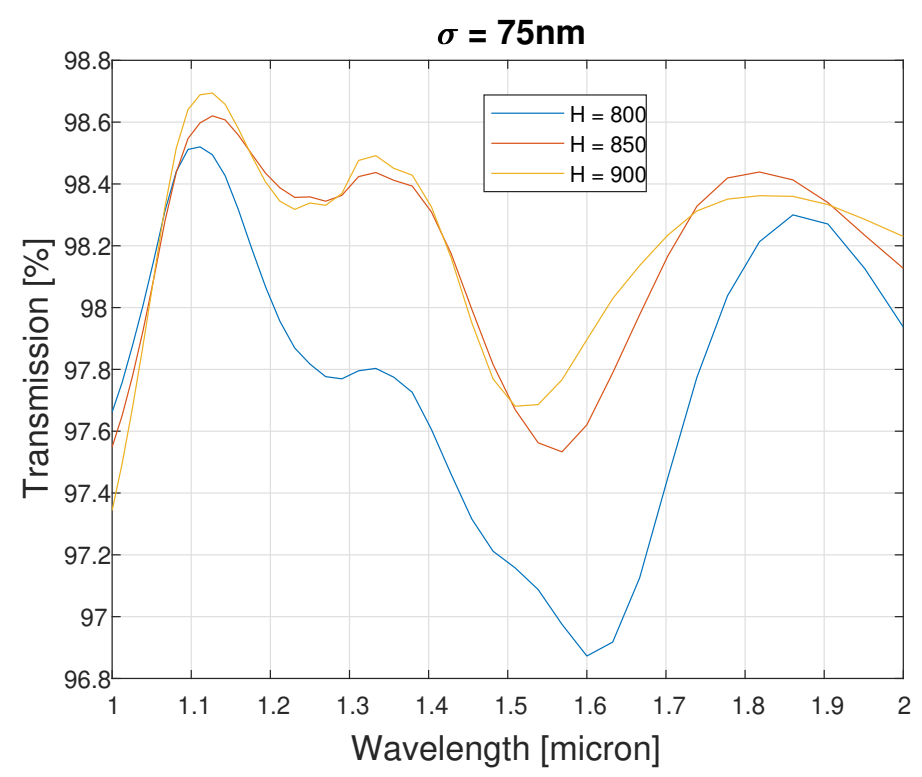

Figure 8: Transmissions for paraboloid facets with $\sigma_{H}=75 \mathrm{~nm}$.

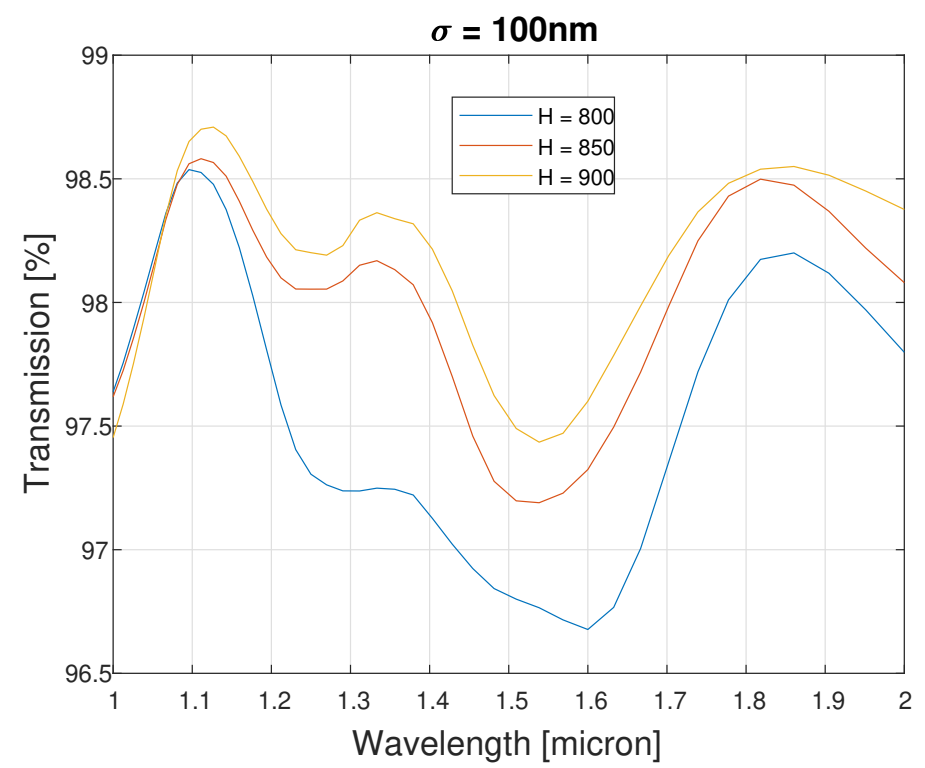

Figure 9: Transmissions for paraboloid facets with $\sigma_{H}=100 \mathrm{~nm}$. 


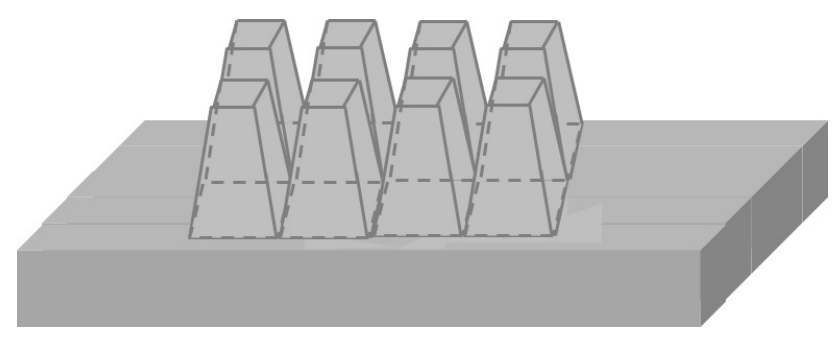

Figure 10: Truncated square pyramidal structures

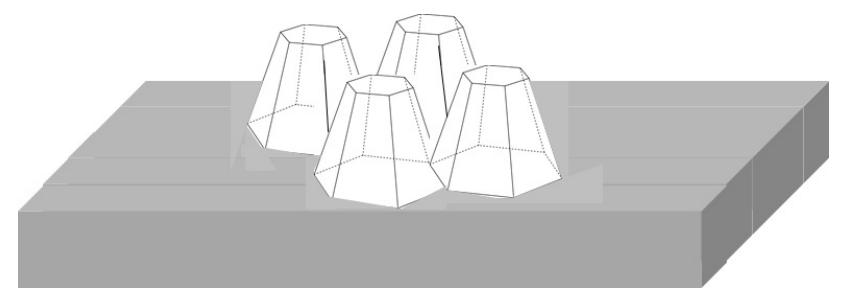

Figure 11: Truncated hex pyramidal structures

\section{Pyramidal structures}

As was already discussed, pyramidal fingers potentially promise an increase in transparency due to improved fill-factor. A better fill- factor we obtain for rectangular (square, for specific facet shapes) pyramids. The illustrations of the structure, based on square and hexagonal pyramids are given in Fig. 10 and Fig 11. The square and hexagonal shapes differs not only by the fill-actor, but also by the scattering shape. Therefore, the final conclusion can be made only after accurate numerical calculations.

The pyramidal structures were considered in different study (e.g., [9]) When using rectangular facets, the main evident advantages of pyramidal structures, is in increasing the fill factor. A facet composed by hex pyramids retains anyway a free area at the edges. For the correct comparison of the efficiency of two structures we have to take their sizes being more or less equivalent (we chose the criterion as equality their heights, and also of their bottom and upper areas). The result, for the example, where the pyramids height $H=900 \mathrm{~nm}$, the bottom size of the surface for the square-pyramid $a_{b}=550 \mathrm{~nm}$, and of the upper square $a_{b}=200 \mathrm{~nm}$, and the same areas for the hex-pyramid are shown in Fig. 12

For easier comparison, we give below the mean values and standard deviations of transmission for the tested pyramids (in natural units) - Trect $_{\text {mean }}=0.976$, and $\sigma_{s} q=0.0035$;

- Thex $x_{\text {mean }}=0.9747$, and $\sigma_{h} x=0.0037$;

The results are slightly different, but give preference to the square pyramid anyway. Therefore, in following, we consider only this type of pyramids.

Actually, adopting a bottom of the pyramidal uni-cell of rectangular and not a square shape, one can achieve the fill-factor as 1 . The results of the proper test are demonstrated in Fig. 13.

Additionally, the base the rectangular pyramids, was taken equal to the base of the square one, deformed for getting the fill factor 1 . The results show the advantage of the proper rectangular structure. Its mean value transmission achieves 0.9 .

However, the choice of the rectangular base dimension is determined by the shape of the facet; thus, it is to be the specific choice. In order not to restrict ourselves by a specific shape of the facet, in following, we analyze mainly square bases of the pyramids. Only one specific case was taken for estimating the effect of maximal fill-factor, and it shows its importance. For this particular facet, the improving factor for the mean transmission is $\eta=1.004$, but the improvement of the standard deviation is significant ((Fig. 14)).

\subsubsection{Truncated and smoothed rectangular pyramids}

Recall, first of all, that dealing with cones ([1]), we got the best results for those with the bottom diameter $d_{b}=450 \mathrm{~nm}$. Therefore, in further analysis, we accept the pyramids with a similar bottom 


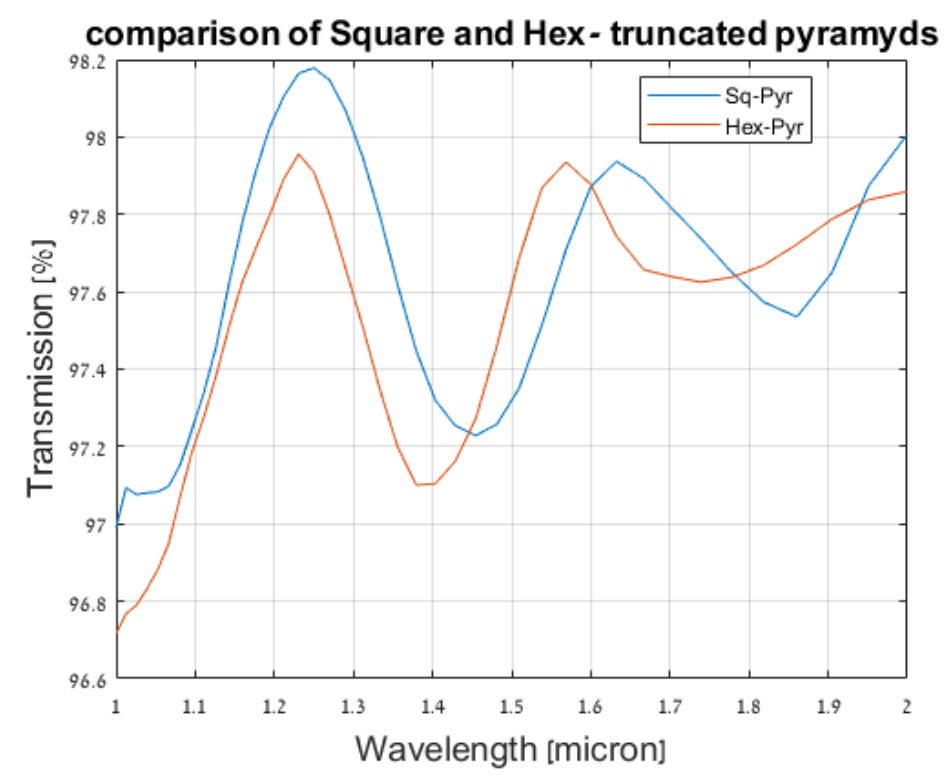

Figure 12: Comparison of transmission of truncated squared and hex pyramids

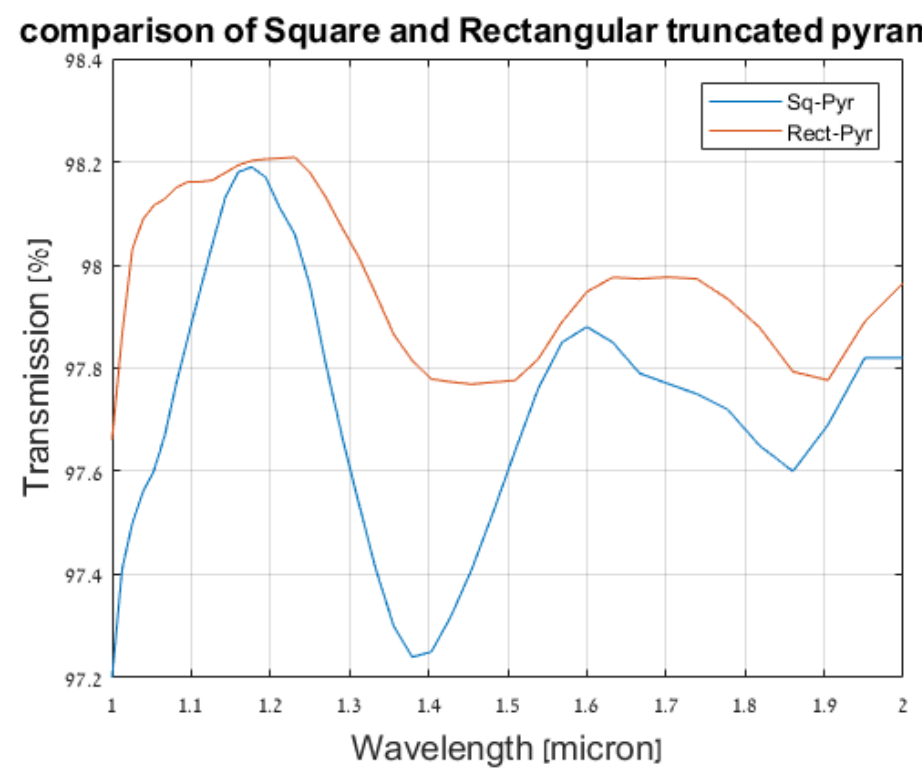

Figure 13: Comparison of transmission of truncated squared and rectangular pyramids 


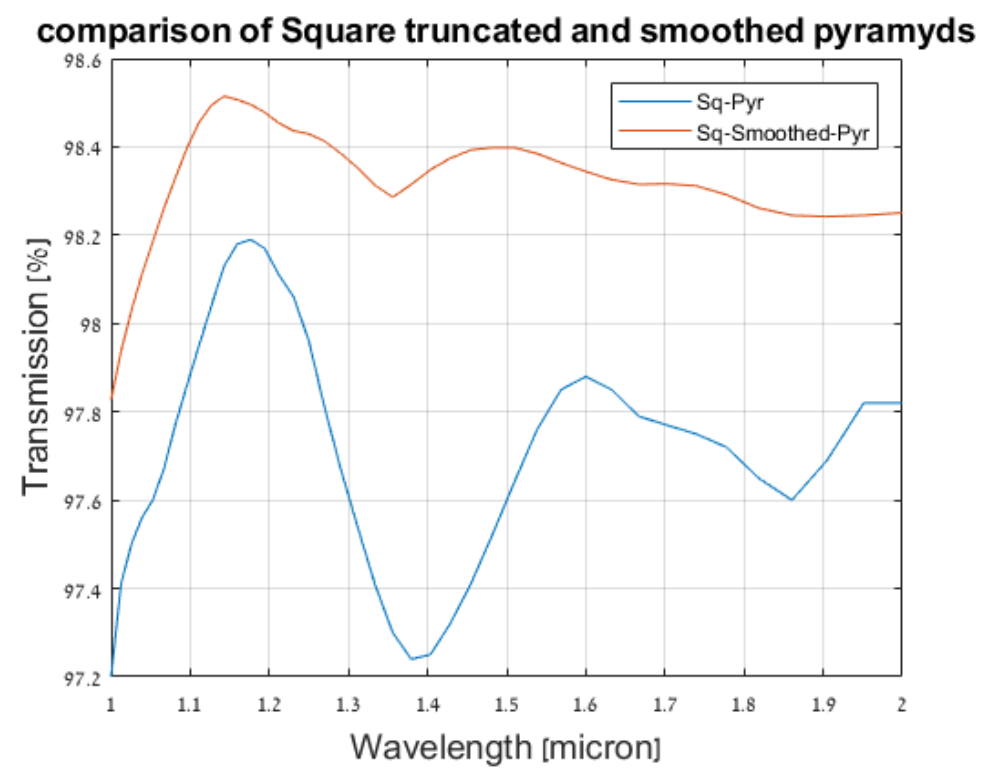

Figure 14: Comparison of transmission of truncated square and smoothed pyramids

area. As previously, we expect some improvement in the transmission by smoothing the pyramids. The height of the parabolic cap (smoothing) is taken as $200 \mathrm{~nm}$. The results of both cases are given in Fig. 14.

The improvement after smoothing is evident. The mean value of transmission achieved a value of 0.983 , with a standard deviation of about 0.0014 . Smoothing of rectangular pyramids is more problematic; however, recalling our previous estimation, one can hope to get the mean value of transmission about $\mathbf{0 . 9 8 7}$. In the next short section, we show the results of randomizing the fingers height on the facet. Another possibility, modification of the edge fingers to smooth the edge effect, is discussed in the Appendix B.

\subsection{Randomization of height of the unit-cells in pyramidal structures}

In this section we like to try the effect of height randomization for the pyramidal structure. The results for different $\sigma_{H}$ are depicted in Fig. 15.

As is seen,the randomization of finger height in the pyramidal structure works even worse than in the conical structure. Moreover, it can give even a negative effect. These results are not so surprising. In literature, we can find an intensive discussion on randomization: pro- and contra (see, e.g., [8]). Such results of randomization for conic structures were discussed above. Thus, we can conclude that any randomization can provide an effect (positive or negative) only for large enough facets.

A numerical analysis of a large facet is a bulky task. A gradient index model for large surfaces (described in [1]) is to be accurate enough in their central area (where edge effects do not contribute). The model works in a range of wavelengths matched to it. Moreover, in this range we do not indicate an essential dependence of the reflectance (transmittance) on the wavelength. Therefore, the need in randomization looks doubtful (see Appendix B in the present work).

\section{From the single facet to the whole waveguide. The simple way for comparing calculation and experimental results}

Underline from the very beginning, that numerical calculation of the reflection and transmission of the wave passed through a waveguide is the very bulky problem. With this, any laboratory measurements (of reflection or transmission characteristics) deal just with a wave transmitted through a one once or several times. However, there is a workaround to almost avoid such problems. 


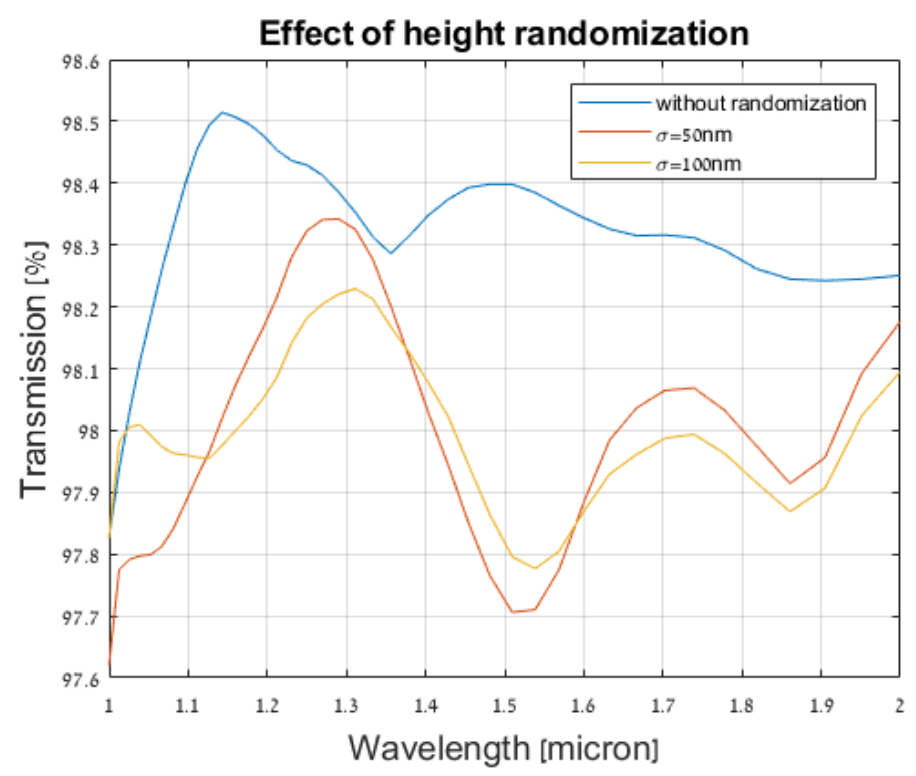

Figure 15: Comparison of transmission of smoothed square pyramids with different randomization of the height

First, we consider a plane wave propagating through a layer (with the refraction index $n$ ) bounded by flat parallel (normal to the direction of wave propagation) boundaries. Let air be the ambient medium (we call this object as layer, or sheet, but not a slab, because of the infinite boundaries).

Let $r$ and $t$ be the reflectance and transmittance of each boundary correspondingly, and $R, T$ the reflectance and transmittance of the layer. Designate the thickness of this sheet as $D$. Let also the input wave amplitude be 1 .

For the reflection from the layer (in the scalar form), we have (see, e.g., [10]).

$$
R=r-\left(r t^{2} e^{i 2 k D}+r^{3} t^{2} e^{i 4 k D}+r^{5} t^{2} e^{i 6 k D}+\ldots\right),
$$

where $k=2 \pi / \lambda$.

In turn, for the transmission though the sheet we write

$$
T=t^{2} e^{i k D}+r^{2} t^{2} e^{i 3 k D}-r^{4} t^{2} e^{i 5 k D}-\ldots
$$

From this equation we get the simple relation

$$
R=r\left(1-T e^{i k D}\right)
$$

Principally, measuring $R$ and $T$, one can estimate the reflectance $r$ of the facet and compare it with the calculated value. However, the single problem in this task is the accurate measurement of $D$ (of the order as $\lambda / 4$ ). On the other hand, we get the like equation while dealing not with the infinite plane but a waveguide mode (for a single-mode waveguide, or the proper superposition of modes for a multi-mode one) with a single difference. Namely, the factor $e^{i k D}$ has to be replaced by another one (designate it as $\Psi$ ).

Thus, factor $\Psi$ occurs the single obstacle for simple experimental testing of our mathematical model.

\section{Discussion and Conclusions}

This study is the continuation of the previous one [1], where we developed the semi-analytic formalism for calculating the parameters of a metasurface made from a high-index dielectric material, similar to that used for the waveguide. Our analysis has demonstrated the transparency effect also to high optical index optical waveguides $(\mathrm{Si})$. Basing on the previous results, we analyze her more cumbersome structures and estimate maximal transmission while using structural surfaces (facets). We considered only those types of surfaces, which actually can be fabricated, therefore, omitting such shape that can 
be defined by polynomial functions of order higher than 2 (or even more complicated). Also, we restricted our analysis by the height of the unit cells achievable for FIB technology, i.e., $d_{b} \leq H / 2$.

The pyramids was introduced, since they can fill rectangular facets more effectively (even achieving the total fill). And despite its more problematic scattering side surface (a ribbing one), numerical calculations actually demonstrate a certain improvement in comparison with cones. Note, that hexagonal base pyramids occur less effective than rectangular one. Again, the base size is determined by the required spectral range and also by the fill-factor. Hexagonal base leave anyway a certain unfilled edge. Smoothing the top has the essential effect for improving the transmission. In turn, the optimally tailored rectangular base improves the transmission additionally.

We showed that this configuration allows to achieve 0.987 transmission level in 1 - 2 micron wavelength range with the flatness. On the contrary, randomization of the heights of the unit cells does not provide a remarkable effect, and for pyramidal structure worsens the transmission. This negative effect can be explained by the small size and specific aspect ratio of the facet.

The surface smallness does not result in transmission degradation determined by the edge effects but restricts the engraving's positive impact.

\section{Author Contributions}

I.G. developed the theoretical aspects and conducted the research. YG built numerical model, run simulations. K.H. and Y.T. run the simulations for different pyramidal structure and rose the idea of using rectangular fingers.

\section{Appendix A: Gradient index model for indefinite surfaces}

Here we turn again to the gradient index model, in order to estimate the result (reflectance, transmittance), which can be considered as the the optimal one, in order to compare it with those obtained with our structures. In [1] we analysed this model for initial choice of the best shape of the fingers. However, one one hand, it is difficult to evaluate directly the integrals appearing there. On the other hand, a computer analysis of multilayered structures ( $M$ sub-layers) fraught to numerical errors growing with adding new layers. And in our case the number of layers is to tend to infinity $(M \rightarrow \infty)$. Because of these reasons, we undertake the analysis of the matrix expression describing an M-layered structure. Let $H$ is the total depth of the gradient index layer. Take all layers be of the same thickness $\delta=H / M$. In the previous publication, we obtained that the best structure for minimal reflectance (if only single scattering is taken into account) is a structure involving fingers (or recesses) with a constant slope. In such a structure, and with compact localization of fingers, the mean refraction index of an effective j-th sub-layer

$$
n_{j}=1+\frac{N-1}{M^{2}} j^{2}
$$

Here we accept that the medium in front of the waveguide possesses the refractive index $n=1$, and the medium behind the waveguide possesses the refractive index $n=N$. With this, if $k$ is the wave factor in vacuum, the effective wave factor in $\mathrm{j}$-th sub-layer is $k_{j}=n_{j} k$.

In follow, we use the matrix-formalism (see, e.g., [10]).

Our basic equation we write as

$$
\left(\begin{array}{c}
E_{\text {in }} \\
E_{R}
\end{array}\right)(T)=\left(\begin{array}{c}
E_{T} \\
0
\end{array}\right)
$$

Transfer matrix $T$ is defined as

$$
T=\left(\begin{array}{cc}
1 & n_{\text {in }} \\
1 & -n_{\text {in }}
\end{array}\right)\left(P_{T}\right)\left(\begin{array}{cc}
1 & 1 \\
1 / n_{\text {out }} & -1 / n_{\text {out }}
\end{array}\right),
$$

where is the product of P-matrices describing the sub-layers.

$$
P_{T}=\prod_{j=1}^{M} P_{j}
$$

and

$$
P_{j}=\left(\begin{array}{cc}
\cos k_{j} \delta & i n_{j} \sin k_{j} \delta \\
i \frac{1}{n_{j}} \sin k_{j} \delta & \cos k_{j} \delta
\end{array}\right)
$$



matrix

Thus, taking into account our agreement concerning ambient media, we have finally for the transfer

$$
T=\left(\begin{array}{ll}
P_{11}+P_{21}+\frac{P_{12}+P_{22}}{N} & P_{11}+P_{21}-\frac{P_{12}+P_{22}}{N} \\
P_{11}-P_{21}+\frac{P_{12}-P_{22}}{N} & P_{12}-P_{21}+\frac{P_{11}-P_{22}}{N}
\end{array}\right)
$$

Now, our equations for scattered (reflected), and transmitted fields obtain form

$$
\begin{gathered}
E_{T}=\frac{1}{T_{11}} \\
E_{R}=T_{21} E_{T}
\end{gathered}
$$

Taking into account that with a large $M$ we have $k \delta<<1$, we are able to make the necessary estimations. Actually, for estimating the fields we need only $T_{11}$ and $T_{21}$.

First of all,

It is easy to notice the following

$$
\begin{gathered}
\cos k_{j} \delta \approx 1-\frac{\delta^{2}}{2}\left(1+\frac{N-1}{M^{2}} j^{2}\right)^{2} \\
\sin k_{j} \delta \approx \delta\left(1+\frac{N-1}{M^{2}} j^{2}\right) .
\end{gathered}
$$

$$
P_{11} \sim \prod_{j=1}^{M}\left[1-\delta_{M}^{2}\left(1+\frac{N-1}{M^{2}} j^{2}\right)\right],
$$

where $\delta_{M}$ denotes $\delta$, for the structure with $M$ layers. The similar estimation can be made also for $P_{22}$.

However, these relations are not detailed enough, for evaluating difference between $P_{11}+P_{22} / N$ and $P_{11}-P_{22} / N$. The main obstacle here is in necessity to multiply finally the matrix $P$ on $\left(\begin{array}{cc}1 & 1 \\ 1 / N & -1 /\end{array}\right)$, while calculate the matrix $T$.

But, since our final purpose is in estimating the structure of $T$, just this is necessary.

For getting the relation in the proper, modify the equation for $P$ in the following manner (call it hear as C-trick).

Introduce the matrices

$$
C_{j}^{+}=\left(\begin{array}{cc}
1 & n_{j} \\
1 & -n_{j}
\end{array}\right)
$$

and

$$
C_{j}^{-}=\left(\begin{array}{cc}
1 & 1 \\
\frac{1}{n_{j}} & -\frac{1}{n_{j}}
\end{array}\right)
$$

These matrices are inverse to each other. Therefore, we can overwrite Eq. A-4 as

$$
P_{T}=C_{1}^{-} C_{1}^{+} P_{1} C_{2}^{-} C_{2}^{+} P_{2} \ldots P_{M} C_{M+1}^{-} C_{M+1}^{+}
$$

Here we take $n_{M+1}=N$. Designate further

$$
T_{j}=C_{j}^{+} P_{j} C_{j+1}^{-}
$$

and then

$$
T_{j}=\left(\begin{array}{cc}
P_{j, 11}+n_{j} P_{j, 21}+\frac{P_{j, 12}+n_{j} P_{j, 22}}{n_{j+1}} & P_{j, 11}+n_{j} P_{j, 21}-\frac{P_{j, 12}+P_{j, 22}}{n_{j+1}} \\
P_{j, 11}-n_{j} P_{j, 21}+\frac{P_{j, 12}-P_{j, 22}}{n_{j+1}} & P_{j, 12}-n_{j} P_{j, 21}+\frac{P_{j, 11}-P_{j, 22}}{n_{j+1}}
\end{array}\right)
$$

In this representation we obtain $T$ in the form, which is simple for estimation.

$$
T=\left(\begin{array}{cc}
1 & 1 \\
1 & -1
\end{array}\right) C_{1}^{-} \prod_{j=1}^{M} T_{j}
$$

For the final estimate, we perform some algebraic transformations. At first, $\frac{n_{j}}{n_{j+1}} \approx 1-j \frac{N-1}{M^{2}} \sim 1-j O\left(\delta_{M}\right)$.

In other words, the difference in the refractive index of neighbour layers is a value of order $O\left(\delta_{M}\right)$. Indeed,

$$
\Delta n=(2 j+1) \frac{N-1}{M^{2}}=(2 j+1) \frac{N-1}{M^{2}} \frac{H}{H}=\delta H(2 j+1) \frac{N-1}{M} \sim \delta .
$$


Now, matrix $T$ obtains a structure.

Note, that since $P_{j, 11}=P_{j, 22}$, after multiplication of these matrices, the relation $P_{11} \approx P_{22}$ with the accuracy of $O\left(\delta_{M}\right)$. With this, we have also $P_{(j, 12)} \sim P_{j, 21} \sim O\left(\delta_{M}\right)$

Now, one can indicate the structure of the matrix T. Rewrite Eq. A-14, in the form

$$
T_{j}=\left(\begin{array}{cc}
2 P_{j, 11}+O\left(\delta_{M}\right) & O\left(\delta_{M}\right) \\
O\left(\delta_{M}\right) & 2 P_{j, 11}+O\left(\delta_{M}\right)
\end{array}\right)
$$

It is easy to see, that the resultant $T$ matrix keeps the similar form. As the last step, we obtain

$$
\left(\begin{array}{cc}
1 & 1 \\
1 & -1
\end{array}\right)\left(\begin{array}{cc}
1 & 1 \\
1 /(1+\Delta n) & -1 /(1+\Delta n)
\end{array}\right)
$$

Recall, that with $M \rightarrow \infty$, we have $\delta_{M} \rightarrow 0$, thus, taking into account Eq. A-5, we obtain that $T$ turns into the unit matrix. For its elements determining the reflected and transmitted waves we have

$T_{11} \rightarrow 1$, and $T_{21} \rightarrow 0$.

It is the result we desired to get. In the field terms, the structure described as the gradient index layer provides its absolute transparency:

$$
E_{T}=E_{\text {in }}
$$

and

$$
E_{R}=0 \text {. }
$$

It is interesting to underline that the wavelength does not appear in our final equation in the explicit form. The physical reasons restrict the range by conditions adopting the model. In the real calculations, the taken wavelength determines the convergence of our infinite products.

\section{Appendix B: The edges effect}

The restriction of the waveguide aperture ever inserts inevitable degradation. Generally, they appear as additional scattering peaks only partly transmitted into the cardinal direction (into the waveguide). In order to analyse the effect approximately in a simple way, we use here the Fourier analysis of the facet structure (this approach is widely known, see, e.g., [11],[12]). By this technique we can probe also the apodization effect (use of FFT for actual calculations see [13]).

The rectangular form of the aperture in our cases, we can restrict ourselves by analyzing its linear cross-section, specifically the short one, represented by the Fourier image.

Take two examples:

- a cross-section of the structure containing three pyramidal fingers;

- a cross-section of the structure containing five paraboloidal fingers.

The next figure presents in comparison the angular diagrams (the angles are calculated in the medium of the waveguide - Si) for these two cases.

One can make the conclusion already from this graph:

1. Indeed, the parabolic shape of the fingers provide concentration of the beam energy in the global lobe.

2. But, actually, this does not matter. For a Si based waveguide (this is just our case), the critical angle for TIR on $\Lambda=1.5 m c$ is $\alpha_{c r} \approx 15.7^{\circ}$. Thus, in our case, the whole diffracted beam inserts in the waveguide without losses.

Try now a structure containing 5 fingers across the narrow side. The angular diagram is shown in Fig. 17. Now, the side-lobes do contribute to the transmitted energy, and still satisfy to the TIR criterion. Again, the parabolic finger shape provides a higher concentration of the beam energy in the main lobe, and yet, it is not critical.

Despite the fact that the previous examples did not indicate any necessity of apodization, we illustrate the effect below basing on the structure involving five triangular fingers. We take for comparison 2 modifications:

1) the perimeter fingers are taken as 1.8 of the basic ones; 2 ) the perimeter fingers are taken as 0.6 of the basic ones.

The results show that apodization does not demonstrate any desired effect. Moreover, the side lobes contain more portion of energy than in the previous tests. This can lead to additional losses for some modified structures. 


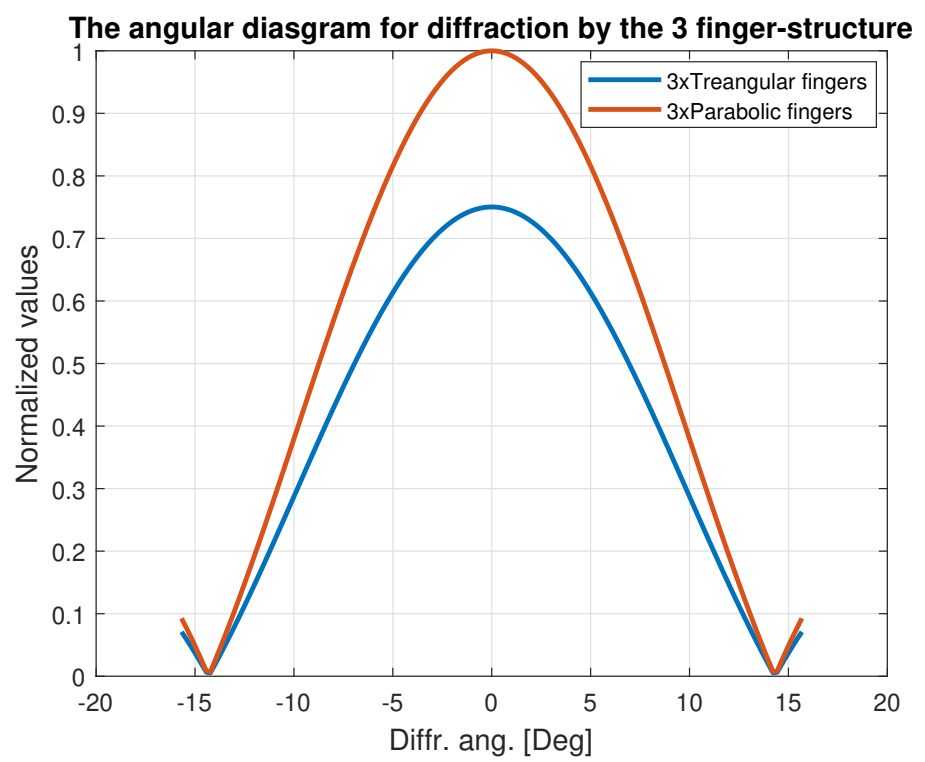

Figure 16: Comparison of the angular diagram (in the proper cross-section) of the forward scattering for the facet containing three fingers at the narrow side: a) blue line - pyramidal fingers, $b$ ) brown line paraboloid fingers

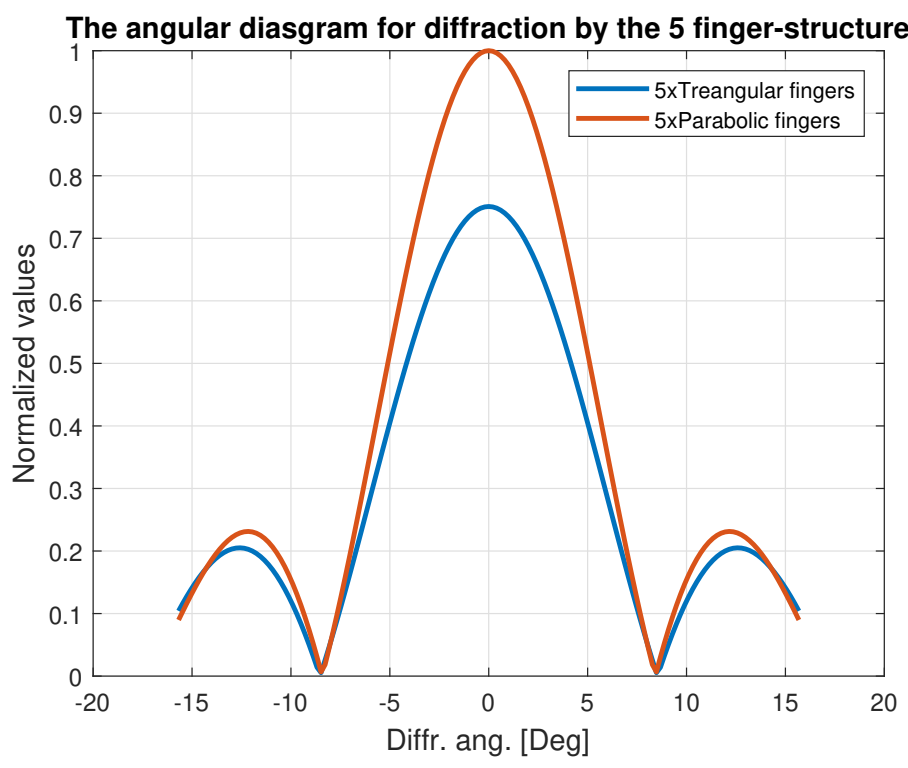

Figure 17: Comparison of the angular diagram (in the proper cross-section) of the forward scattering for the facet containing five fingers at the narrow side: a) blue line - pyramidal fingers, b) brown line paraboloid fingers 


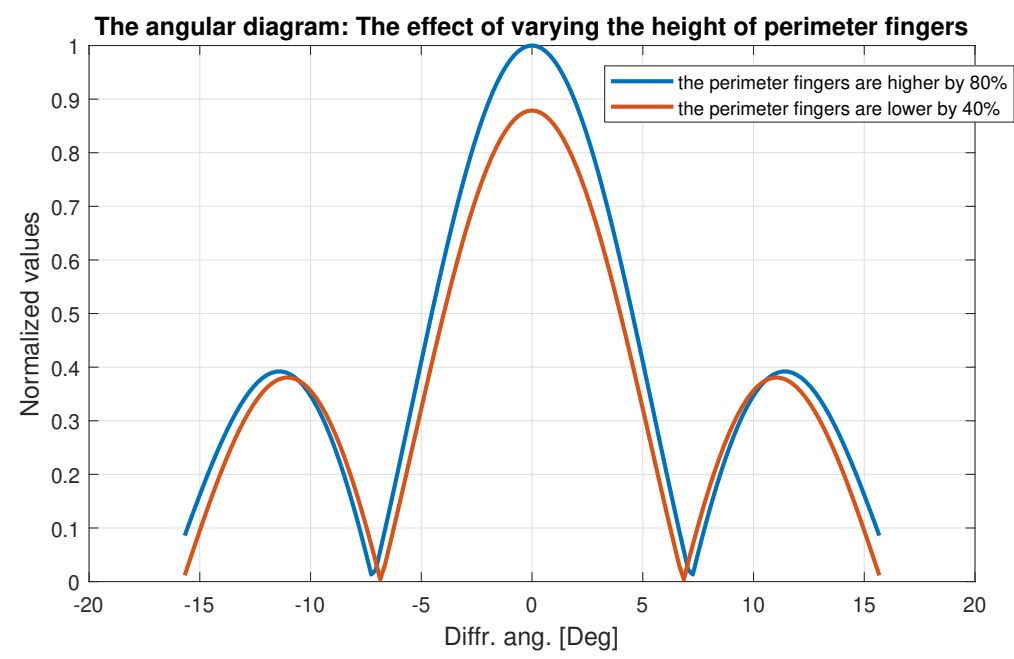

Figure 18: Comparison of the angular diagram (in the proper cross-section) of the forward scattering for the facet containing five fingers at the narrow side: a) blue line - higher perimeter fingers, $b$ ) brown line - lower perimeter fingers

\section{References}

[1] A. Karabchevsky, E. Falek, Y. Greenberg, M. Elman, Y. Keren, and I. Gurwich, “Broadband transparency with all-dielectric metasurfaces engraved on waveguide facets: effect of inverted and extruded features based on babinet's principle," Nanoscale Advances, 2020.

[2] A. Deinega, I. Valuev, B. Potapkin, and Y. Lozovik, "Minimizing light reflection from dielectric textured surfaces," JOSA A, vol. 28, no. 5, pp. 770-777, 2011.

[3] J. H. Rosen, "Diffraction patterns produced by focused laser beams," tech. rep., RAND CORP SANTA MONICA CA, 1972.

[4] F. Simonetti, M. Fleming, and E. A. Marengo, "Illustration of the role of multiple scattering in subwavelength imaging from far-field measurements," JOSA A, vol. 25, no. 2, pp. 292-303, 2008.

[5] E. Garnett and P. Yang, "Light trapping in silicon nanowire solar cells," Nano letters, vol. 10, no. 3, pp. 1082-1087, 2010.

[6] F. Simonetti, "Multiple scattering: The key to unravel the subwavelength world from the far-field pattern of a scattered wave," Physical Review E, vol. 73, no. 3, p. 036619, 2006.

[7] R. H. Siddique, G. Gomard, and H. Hölscher, "The role of random nanostructures for the omnidirectional anti-reflection properties of the glasswing butterfly," Nature communications, vol. 6, p. 6909, 2015.

[8] G. Berginc, "Periodic or random nanostructures for light scattering control," Applied Physics A, vol. 122, no. 4, p. 318, 2016.

[9] D. H. Raguin and G. M. Morris, "Antireflection structured surfaces for the infrared spectral region," Applied optics, vol. 32, no. 7, pp. 1154-1167, 1993.

[10] M. Born and E. Wolf, Principles of optics: electromagnetic theory of propagation, interference and diffraction of light. Elsevier, 2013.

[11] L. Novotny and B. Hecht, Principles of nano-optics. Cambridge university press, 2012.

[12] T. Phillips, "X-ray crystallography and the fourier transform," American Mathematical Society, http://www. ams. org/samplings/feature-column/fc-2011-10, accessed April, 2017.

[13] D. Mas, J. Garcia, C. Ferreira, L. M. Bernardo, and F. Marinho, “Fast algorithms for free-space diffraction patterns calculation," Optics communications, vol. 164, no. 4-6, pp. 233-245, 1999. 